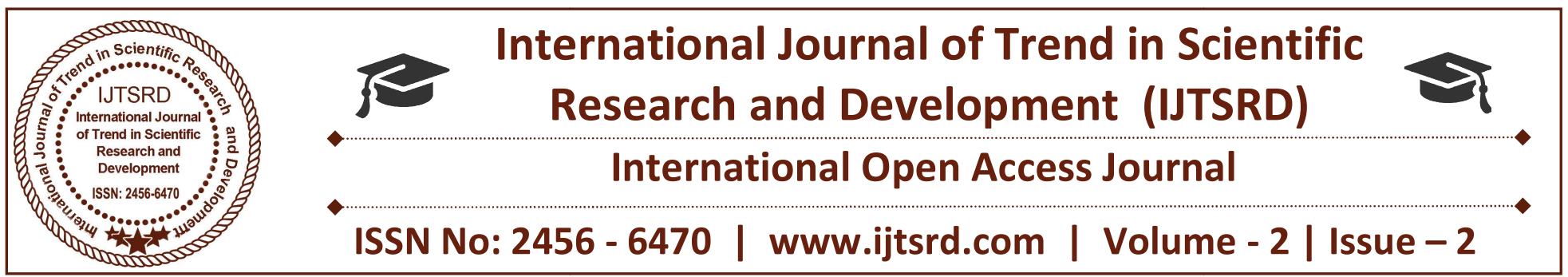

\title{
Security System for Car using CAN Protocol
}

\section{Poonam Bedarkar}

Department of Electronics Engineering, Kavikulguru Institute of Technology and Science

Ramtek, Nagpur, Maharashtra, India

\author{
Atul Halmare \\ Information Technology Department \\ Kavikulguru Institute of Technology and Science \\ Ramtek, Nagpur, Maharashtra, India
}

\begin{abstract}
The design consist of a central "Airbag control unit"(ACU) controls a number of related sensors within the vehicle like accelerometers, impact sensors, wheel speed sensors, gyroscopes, brake pressure sensors, and seat occupancy sensors. When the requisite 'threshold' has been reached or exceeded, the airbag control unit will trigger the ignition of a gas generator propellant to rapidly inflate a nylon fabric bag. The inflated airbag reduces the deceleration experienced by the passenger during the crash through the process of venting gas out of small vent holes in a controlled manner absorbing the energy of the occupant impacting the bag. CAN is a serial communication protocol. The CAN bus may be used in vehicles to connect engine control unit and transmission, or (on a different bus) to connect the door locks, climate control, seat control.
\end{abstract}

Keywords: CAN Bus protocol, MEMS, Microcontroller, MPLAB software

\section{INTRODUCTION}

Since the early 1940 's, automakers have continually improved their vehicles' technology by integrating an increasing amount of electronic components.

As technology progressed, the vehicles became more complex as electronic components replaced mechanical systems and provided additional comforts, convenience, and safety features.

Up until the release of CAN Bus, vehicles contained enormous amounts of wiring which was necessary to interconnect all of the various electronic components.

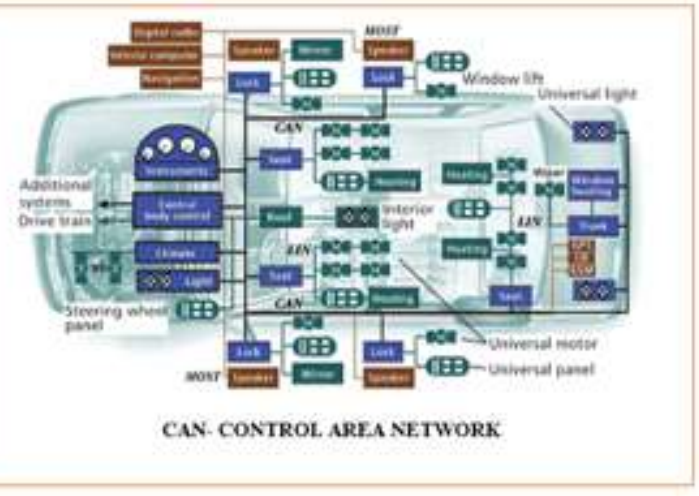

Fig 1.1: Control area network

Due to the vast amount of wiring, an aftermarket installation requires the installer not only to understand how the integrated systems communicate with each other, but also requires numerous connections to be made throughout the vehicle. To make matters worse, the vehicle wiring differs between vehicle years, makes, and even models.

As a result, installers need to be highly knowledgeable and perform intensive labor for the most trivial after market equipment or the installation shop experiences countless hours of lost time on troubleshooting and sometimes even expensive claims for damaged OEM equipment.

An airbag is part of a vehicle's safety restraint system, a flexible envelope designed for rapid inflation in an automobile collision, to prevent vehicle occupants from striking hard interior objects such as steering wheels. It is considered a "passive" safety component not requiring any input or action from the user, rather than an "active" component — such as a seat belt, 
which requires fastening. "Airbag control unit"(ACU) has a number of related sensors inside the vehicle such as accelerometers, impact sensors, wheel speed sensors, gyroscopes, brake pressure sensors, and seat occupancy sensors.

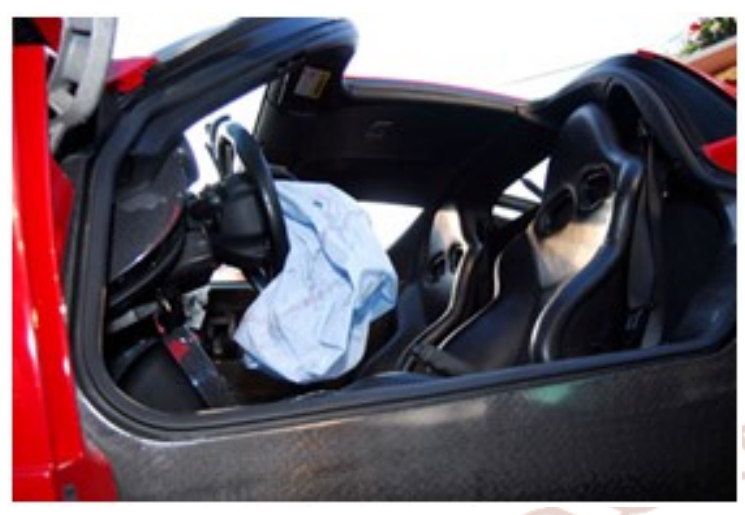

Fig 1.2: The deployed air bag in car

\section{SYSTEM MODEL}

\subsection{Designed System}

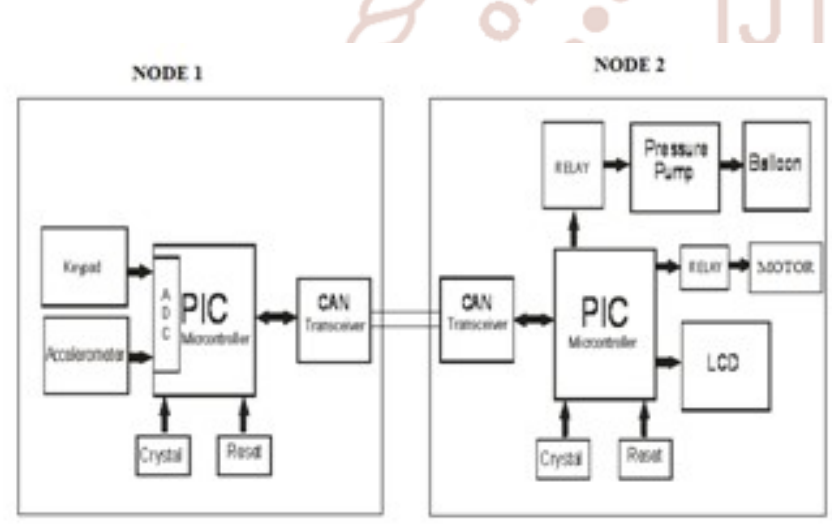

Fig. 2.1.1: Block diagram

In this there are two nodes. The one node is connected to the accelerometer and keypad. The other node would be connected to the relay. The PIC microcontroller would be used for the Node 1 and Node two. The MCP2551 transceiver would be used as CAN transceiver. The two nodes would be connected on the CAN bus.

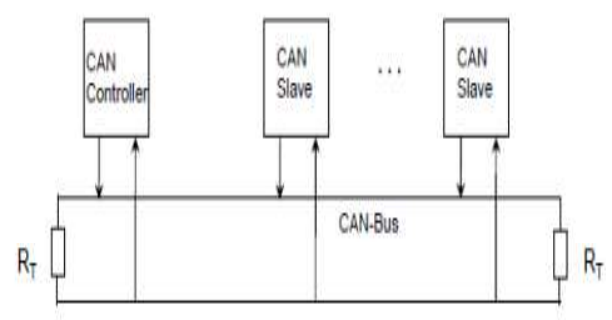

Fig 2.1.2: CAN Transmission
In this project there would be two sections Node1 and Node2. The Node1 part would contain the MEMS sensors. The sensor output would be connected to the PIC microcontroller. The PIC would be connected to the CAN transceiver.

The Node 2 would contain the graphics LCD would display the sensor values. The Node 2 would also be connected to the relay which will control the pump.

Whenever the sensor data will exceed the set valve the will be send on the CAN bus and will trigger the relay and on the pump.

\subsection{CAN PROTOCOL OVERVIEW:}

CAN mean Controller Area Network. CAN is an asynchronous serial communication protocol. Multi master concept: CSMA/ CA (Carrier Sense Multiple Access / Collision Avoidance), Message priority.

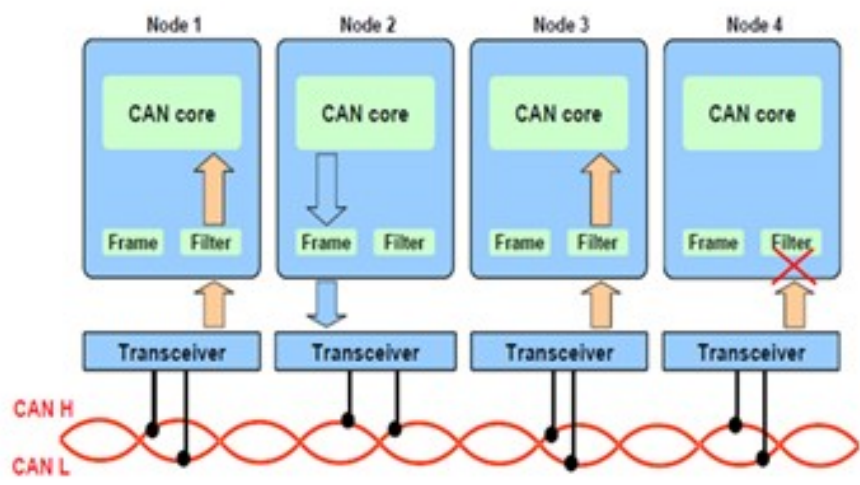

Fig 2.2.1: Bus Topology

\subsubsection{BEFORE CAN BUS:}

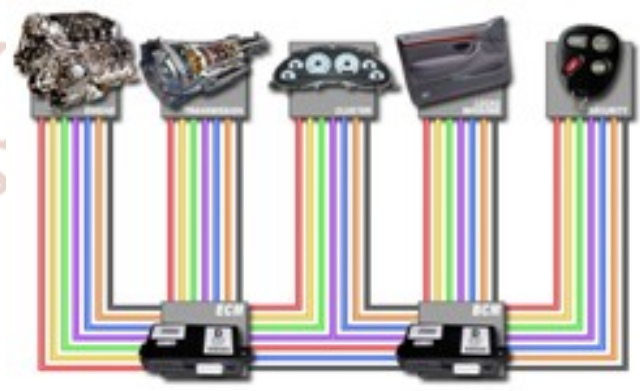

Fig 2.2.2: Before CAN Bus

Due to the vast amount of wiring, an after market installation requires the installer not only to understand how the integrated systems communicate with each other, but also requires numerous connections to be made throughout the vehicle. To make matters worse, the vehicle wiring differs between vehicle years, makes, and even models. As a 
result, installers need to be highly knowledgeable and perform intensive labor for the most trivial after market equipment or the installation shop experiences countless hours of lost time on troubleshooting.

\section{2.2 AFTER CAN BUS:}

By reducing the vehicles wiring by $2 \mathrm{~km}$, the vehicles overall weight was significantly reduced by at least $50 \mathrm{~kg}$ and using only half the connectors. For the first time, each of the vehicles systems and sensors were able to communicate at very high speeds $(25 \mathrm{kbps}-$ $1 \mathrm{Mbps}$ ) on a single or dual-wire communication line as opposed to the previous multi-wire looms. However, the introduction of CAN Bus also increased the vehicles complexity and made after market installations even more difficult and in many cases impossible to perform.

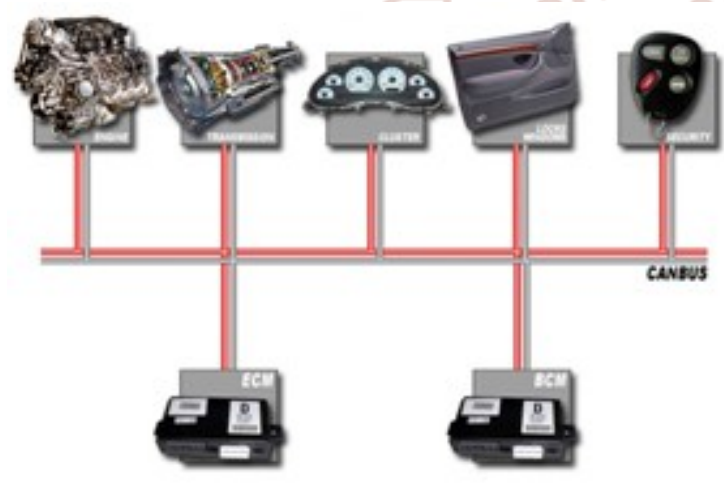

Fig 2.2.3: After CAN Bus

\subsubsection{CAN LAYERS:}

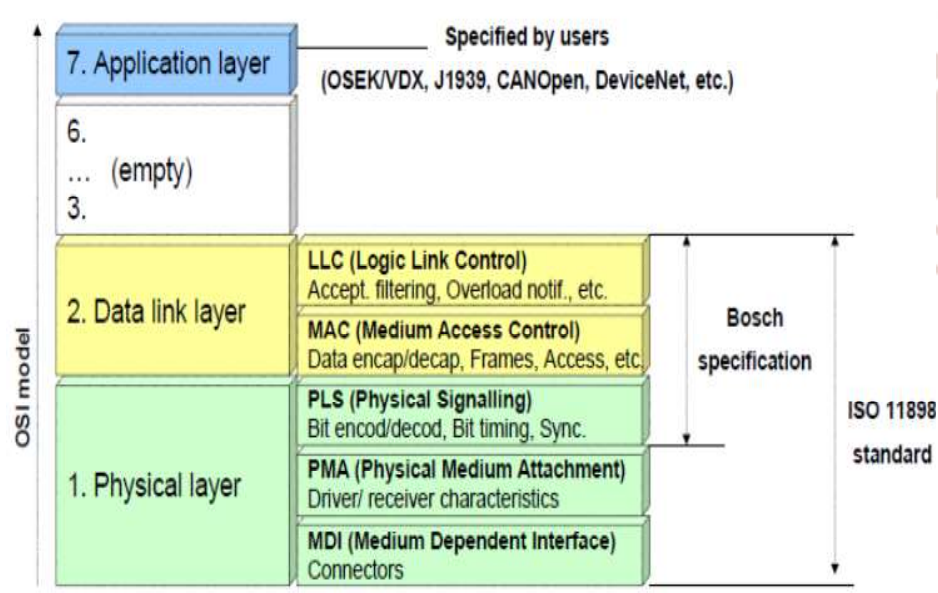

Table 2.2.4: CAN Layers

\subsection{PIC Peripheral Interface controller}

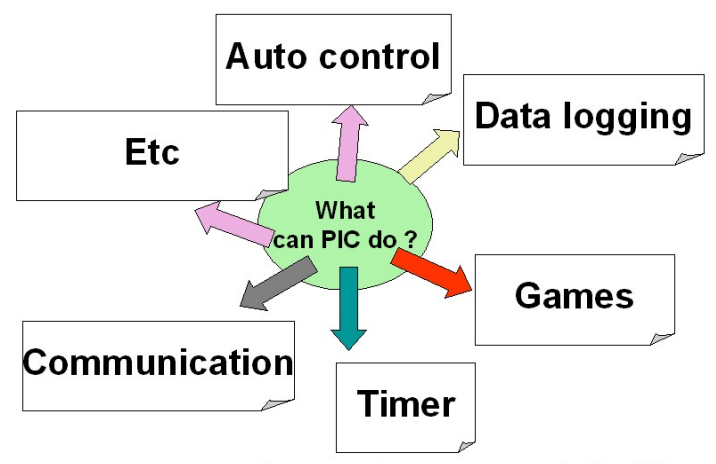

Fig 2.3.1: Applications of PIC

PIC $18 F 4685$ is 40 pin Enhanced Flash Microcontrollers with ECA Technology,10-Bit A/D and nano Watt Technology designated with an " $F$ " in the part number (such as PIC18F2685), accommodate an operating VDD range of $4.2 \mathrm{~V}$ to $5.5 \mathrm{~V}$.

\subsection{Airbag Systems in Automobiles:}

An airbag is part of a vehicle's safety restraint system, a flexible envelope designed for rapid inflation in an automobile collision, to prevent vehicle occupants from striking hard interior objects such as steering wheels. It is considered a "passive" safety component not requiring any input or action from the user, rather than an "active" component - such as a seat belt, which requires fastening.

\subsubsection{Airbag Inflation}

When the frontal part of airbags deployed, a signal will be sent to the inflator unit within the control unit of airbag system. An igniter will start with a rapid chemical reaction generating primarily nitrogen gas $\left(\mathrm{N}_{2}\right)$ which will fill the airbag making it to deploy through the module cover.

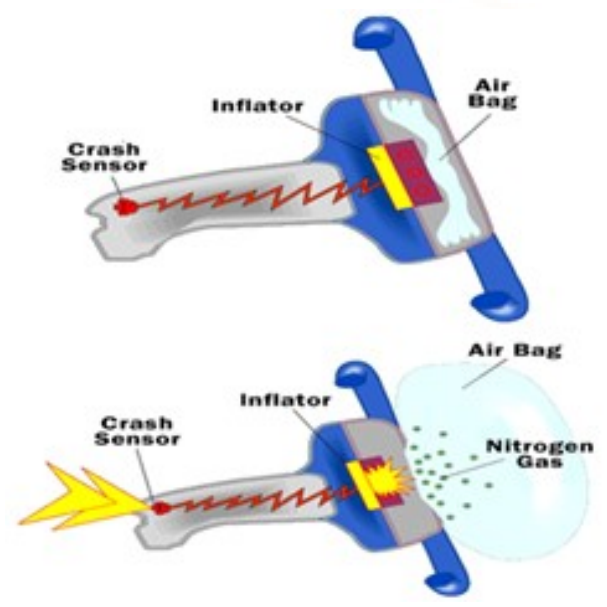

Fig 2.4.1: Inflation of airbag 


\subsection{MEMS}

Micro-Electro-Mechanical Systems (MEMS) is the integration of mechanical elements, sensors, actuators, and electronics on a common silicon substrate through micro fabrication technology. While the electronics are fabricated using integrated circuit (IC) process sequences (e.g., CMOS, Bipolar, or BICMOS processes), the micromechanical components are fabricated using compatible "micromachining" processes that selectively etch away parts of the silicon wafer or add new structural layers to form the mechanical and electromechanical devices.

\section{IMPLEMENTATION}

\subsection{MPLAB:}

MPLAB IDE is a software program that runs on a PC to develop applications for Microchip microcontrollers. It is Integrated Development Environment (IDE) as it provides a single integrated "environment" for developing a code for embedded microcontrollers. Embedded systems development and how MPLAB IDE is used.

MPLAB (IDE) is a free and integrated toolset for developing embedded applications like Microchip's and microcontrollers. MPLAB IDE runs as a 32-bit application on MS Windows ${ }^{\circledR}$, is easy to use and includes a host of free software components for fast application development and super-charged debugging. MPLAB IDE is unified graphical user interface for additional Microchip and third party software and hardware development tools. Moving between tools is a snap, and upgrading from the free software simulator to hardware debug and programming tools is done in a flash as MPLAB has the same user interface for all tools.

\subsection{MPLAB IDE FEATURES:}

\section{A] Flexible customizable programmer's text editor:}

1 Fully integrated debugging with right mouse click menus for breakpoints, trace and editor functions.

2 Tabbed editor option or separate source windows.

3 Recordable macros.

4 Context sensitive color highlighting for assembly, $\mathrm{C}$ and BASIC code readability.

5 Mouse over variable to instantly evaluate the contents of variables and registers.
6 Set breakpoints and trace points directly in editor to instantly make changes and evaluate their effects.

7 Graphical project manager.

8 Version control support for MS Source Safe, CVS, PVCS, Subversion.

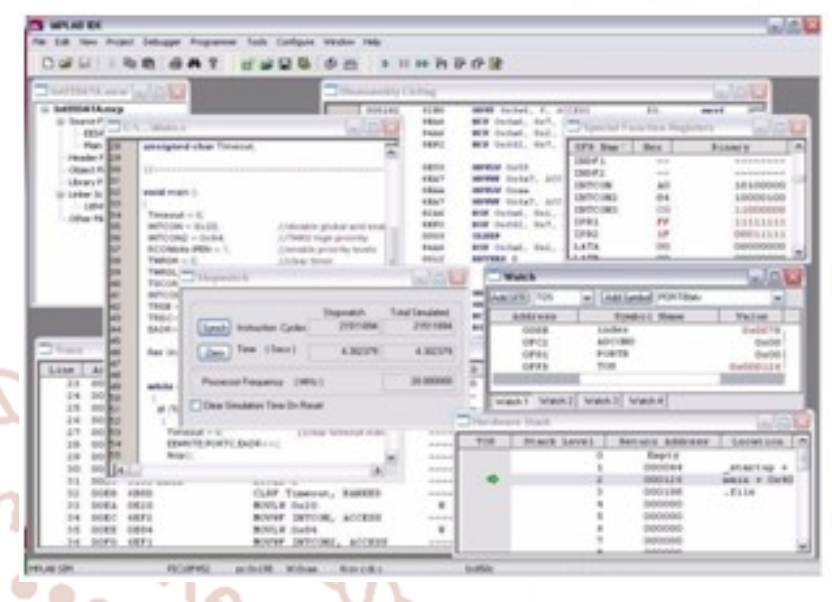

Fig 3.2.1: MPLAB software view

\section{Conclusion}

CAN uses its bandwidth efficiently and the access to the Bus is organized according to the priorities of the individual modules. The successor of the CAN Bus will be Flex ray, which has in addition Time -and Frequency- Mux capabilities. But CAN will coexist for at least the next one or two decade. Therefore it is very suitable for applications where real-time capabilities are needed.

\section{ACKNOWLEDGEMENT}

We sincerely like to thank all the people who have directly and indirectly encouraged us and helped us in working out our research. Also a big thank to K.I.T.S. College from where we got complete support.

\section{REFERENCES}

[1] Huaqun Guo, lun lie Ang, and Yongdong Wu, "Extracting Controller Area Network Data for Reliable Car Communication", (2010 February)

[2] Heffernan D. \& Leen G., "ICT based research at Limerick contributes to automotive 'drive-bywire' technology", (2008 September).

[3] Kumar, M. A.Verma and A. Srividya, Response Time "Modeling of Controller Area Network (CAN). Distributed Computing and Networking, 
Lecture Notes in Computer Science Volume 5408, [10] Manjunath, T K N Maheswari, Andrews Samraj, p 163-174, 2009.

[4] Tindell, K., A. Burns, and A.J. Wellings, Calculating controller area network (CAN) message response times. Control Engineering Practice, 3(8): p. 1163-1169, 2005.

[5] Li, M., Design of Embedded Remote Temperature Monitoring System based on Advanced RISC Machine. Electrotechnics Electric, 06, p. 273, 2009.

[6] Prodanov, W., M. Valle, and R. Buzas, A controller area network bus transceiver behavioral model for network design and simulation. IEEE Transactions on Industrial Electronics, 56(9): p. 3762-377, 2009.

[7] ISO (1993). Road Vehicles: Interchange of Digital Information: Controller Area Network (CAN) for High Speed Communication. ISO 11898:1993.

[8] B.Gmbh, "CAN specification” vol 1 Version 2.0, 1991.

[9] Microchip Technology, Inc. (2007). PIC 18F2455/2550/4455/4550 Data Sheet. Sharmila, Chidaravalli (2013) "Locking and Unlocking of Theft Vehicles, Using CAN", Proceedings of 2013 International Conference on Green High Performance Computing, March 2013

[11] R. Ramani, S. Valarmathy, N.Suthanthira, Vanitha, S.Selvaraju (2013). "Vehicle Tracking and Locking System Based on GSM and GPS" I.J. Intelligent Systems and Applications, 2013, 09, pp 86-93, August 2013

[12] Vinoth Kumar Sadagopan Upendran Rajendran Albert Joe Francis (2011)" Antitheft control System Design Using Embedded System,'IEEE, 2011

[13] B.G. Nagaraja, Ravi Rayappa, M Mahesh, Chandrasekhar M Patil, Dr. T.C. Manjunath,"Design \& Development of a GSM Based Vehicle Theft Control System", International Conference on Advanced Computer Control, DOI 10.1102009 pp 148-154

[14] Ruchita J. Shah, Anuradha P. Gharge, "GSM Based Car Security System", International Journal of Engineering and Innovative Technology, Volume 2, Issue 4, pp 203-206,October 2012 